\title{
MARC DELÉGLISE
}

\section{Encadrement de la fonction sommatoire des inverses des termes d'une progression arithmétique}

Annales de la faculté des sciences de Toulouse $6^{e}$ série, tome $5, \mathrm{n}^{\circ} 3$ (1996), p. 471-492

<http://www.numdam.org/item?id=AFST_1996_6_5_3_471_0>

(C) Université Paul Sabatier, 1996, tous droits réservés.

L'accès aux archives de la revue «Annales de la faculté des sciences de Toulouse » (http://picard.ups-tlse.fr/ annales/) implique l'accord avec les conditions générales d'utilisation (http://www.numdam.org/conditions). Toute utilisation commerciale ou impression systématique est constitutive d'une infraction pénale. Toute copie ou impression de ce fichier doit contenir la présente mention de copyright.

\section{Numdam}

Article numérisé dans le cadre du programme Numérisation de documents anciens mathématiques http://www.numdam.org/ 


\title{
Encadrement de la fonction sommatoire des inverses des termes d'une progression arithmétique ${ }^{(*)}$
}

\author{
MARC DeLÉGLISE ${ }^{(1)}$
}

RÉSUMÉ. - Soient $a, b, x>0$. Nous écrivons

$$
\begin{aligned}
& A_{a, b}(x)=\sum_{0<u \leq x}^{u \equiv a \bmod b} \frac{1}{u}-\sum_{0<u<a}^{u \equiv a \bmod b} \frac{1}{u} \quad\left(=\sum_{n<x} \frac{1}{n} \quad \text { lorsque } a=b=1\right) \\
& q_{a, b}(x)=\frac{\{(x-a) / b\}-1 / 2}{x}, \quad \gamma_{a, b}=\frac{1}{a}-\frac{\ln a}{b}-\int_{a}^{\infty} \frac{\{(u-a) / b\}}{u^{2}} \mathrm{~d} u .
\end{aligned}
$$

Nous prouvons

$$
A_{a, b}(x)=\frac{\ln x}{b}+\gamma_{a, b}-q_{a, b}(x)+J_{a, b}(x)
$$

avec

$$
J_{a, b}(x)=\int_{x}^{\infty} \frac{\{(u-a) / b\}-1 / 2}{u^{2}} \mathrm{~d} u,
$$

et les inégalités, vérifiées pour tout $a, b, x>0$,

$$
-\frac{b}{12 x^{2}} \leq J_{a, b}(x) \leq \frac{b}{24 x^{2}} .
$$

Abstract. - Let $a, b, x>0$. We write

$$
\begin{gathered}
A_{a, b}(x)=\sum_{0<u \leq x}^{u \equiv a \bmod b} \frac{1}{u}-\sum_{0<u<a}^{u \equiv a \bmod b} \frac{1}{u} \quad\left(=\sum_{n<x} \frac{1}{n} \text { when } a=b=1\right) \\
q_{a, b}(x)=\frac{\{(x-a) / b\}-1 / 2}{x}, \quad \gamma_{a, b}=\frac{1}{a}-\frac{\ln a}{b}-\int_{a}^{\infty} \frac{\{(u-a) / b\}}{u^{2}} \mathrm{~d} u .
\end{gathered}
$$

(*) Reçu le $1^{\text {er }}$ juin 1994

(1) Département de Mathématiques, Université Lyon I, 43 boulevard du 11 Novembre 1918, F-69622 Villeurbanne Cedex (France) 
We prove

$$
A_{a, b}(x)=\frac{\ln x}{b}+\gamma_{a, b}-q_{a, b}(x)+J_{a, b}(x)
$$

with

$$
J_{a, b}(x)=\int_{x}^{\infty} \frac{\{(u-a) / b\}-1 / 2}{u^{2}} \mathrm{~d} u,
$$

and the inequalities, true for all $a, b, x>0$,

$$
-\frac{b}{12 x^{2}} \leq J_{a, b}(x) \leq \frac{b}{24 x^{2}} .
$$

\section{Introduction}

On sait que $A(n)=\sum_{k=1}^{n} 1 / k$ admet un développement asymptotique dont les premiers termes sont

$$
A(n)=\ln (n)+\gamma+\frac{1}{2 n}-\frac{1}{12 n^{2}}+\frac{1}{120 n^{4}}+\mathrm{O}\left(\frac{1}{n^{6}}\right)
$$

où $\gamma=0,577, \ldots$ est la constante d'Euler. Ce développement peut être obtenu de diverses façons, en particulier par la formule sommatoire d'Euler MacLaurin. Il en résulte que

$$
-\frac{1}{12 n^{2}} \leq A(n)-\ln n-\gamma-\frac{1}{2 n} \leq 0 .
$$

Dans la pratique on est parfois amené à considérer la somme

$$
A(x)=\sum_{n \leq x} \frac{1}{n}
$$

$x$ étant un nombre réel positif quelconque dont on ne connaît pas la partie entière. Il est alors utile d'obtenir un encadrement de $A(x)$. On se propose de prouver ici qu'en remplaçant le terme $-1 / 2 n$ qui figure dans (1) par le terme $(\{x\}-1 / 2) / x$, qui se réduit à $-1 / 2 x$ quand $x$ est entier, on obtient encore un encadrement de $A(x)$ de précision $\mathrm{O}\left(1 / x^{2}\right)$, plus précisément :

$$
-\frac{1}{12 x^{2}} \leq A(x)-\ln x-\gamma+\frac{\{x\}-1 / 2}{x} \leq \frac{1}{24 x^{2}}
$$

pour tout $x$ positif. Il est assez naturel d'élargir un peu le problème et de se demander ce qui se passe si on remplace la suite des entiers naturels par la progression arithmétique $(a+b n)_{n \in N}, a, b \geq 0$. 
Fonction sommatoire des inverses des termes d'une progression arithmétique

Pour tous $a, b, x$ réels $>0$ on pose donc

$$
\begin{aligned}
A_{a, b}(x)=\sum_{0<u \leq x}^{u \equiv a \bmod b} \frac{1}{u}-\sum_{0<u<a}^{u \equiv a \bmod b} \frac{1}{u}( & \left.=\sum_{a \leq u \leq x}^{u \equiv a \bmod b} \frac{1}{u} \quad \text { si } x \geq a\right) \\
& \left(=-\sum_{x<u<a}^{u \equiv a \bmod b} \frac{1}{u} \text { si } x<a\right) .
\end{aligned}
$$

L'objet de ce travail est la démonstration de la proposition 1 et du théorème 2 ci-dessous. Dans le cas $a=b=1$, ces deux énoncés contiennent (2). Nous nous attendions à trouver une démonstration assez simple du théorème 1; nous n'y sommes pas parvenus; la démonstration obtenue est assez calculatoire, et nous avons effectué les calculs en utilisant l'outil de calcul formel Maple.

Les constantes $\gamma_{a, b}$ introduites dans la proposition 1 sont étroitement liées à la fonction $\Psi$, dérivée logarithmique de la fonction $\Gamma$. On a en effet $\gamma_{a, b}=-(1 / a) \Psi(a / b)$. On pourra trouver dans [2] des propriétés des constantes d'Euler $\gamma_{a, b}$ pour $a, b$ entiers. En particulier on y donne une démonstration élémentaire de la formule de Gauss qui donne les valeurs en les points rationnels de la fonction $\Psi$

$$
\Psi\left(\frac{a}{b}\right)=-\gamma-\ln \left(\frac{b}{2}\right)-\frac{\pi}{2} \operatorname{cotg}\left(\frac{\pi a}{b}\right)+2 \sum_{0<j \leq k / 2} \cos \frac{2 \pi j a}{b} \ln \sin \frac{\pi j}{b} .
$$

Proposition 1.- On note $\lfloor x\rfloor$ la partie entière de $x$ et par $\{x\}$ sa partie fractionnaire, c'est- $\grave{a}$-dire $\{x\}=x-\lfloor x\rfloor$. On a

$$
A_{a, b}(x)=\frac{\ln x}{b}+\gamma_{a, b}-q_{a, b}(x)+J_{a, b}(x)
$$

en posant

$$
\begin{aligned}
& \gamma_{a, b}=\frac{1}{a}-\frac{\ln a}{b}-\int_{a}^{+\infty} \frac{\{(u-a) / b\}}{u^{2}} \mathrm{~d} u \\
& q_{a, b}(x)=\frac{\{(x-a) / b\}-1 / 2}{x} \\
& J_{a, b}(x)=\int_{x}^{+\infty} \frac{\{(u-a) / b\}-1 / 2}{u^{2}} \mathrm{~d} u .
\end{aligned}
$$


ThÉORÈme 1. - Pour tous $a, b, x>0$ on a l'encadrement

$$
-\frac{b}{12 x^{2}} \leq J_{a, b}(x) \leq \frac{b}{24 x^{2}}
$$

et les coefficients de $b / x^{2}$ dans cet encadrement sont optimaux.

\section{Rappels sur les polynômes et les fonctions de Bernoulli}

Soit $\mathrm{P}_{k}$ la suite des polynômes de Bernoulli :

$$
\begin{gathered}
\mathrm{P}_{1}(u)=u-\frac{1}{2}, \quad \mathrm{P}_{2}(u)=u^{2}-u+\frac{1}{6}, \quad \mathrm{P}_{3}(u)=u^{3}-\frac{3}{2} u^{2}+\frac{1}{2} u \\
\mathrm{P}_{4}(u)=u^{4}-2 u^{3}+u^{2}-\frac{1}{30}, \quad \mathrm{P}_{5}(u)=u^{5}-\frac{5}{2} u^{4}+\frac{5}{3} u^{3}-\frac{1}{6} u
\end{gathered}
$$

et $t \mapsto P_{k}(t)$ la suite des fonctions de Bernoulli définies par $P_{k}(t)=\mathrm{P}_{k}(\{t\})$. On notera aussi $B_{k}$ le $k^{\text {ième }}$ nombre de Bernoulli, c'est-à-dire $B_{k}=\left|\mathcal{P}_{2 k}(0)\right|$. On a $B_{1}=1 / 6$ et $B_{2}=1 / 30$. On rappelle sous forme de lemme les propriétés des fonctions de Bernoulli qu'on utilisera dans la suite. On pourra consulter par exemple [1].

LEMME 1

1) Sur chaque intervalle ouvert $] n, n+1\left[, P_{k}\right.$ est dérivable, de dérivée $k P_{k-1} ;$ pour $k \geq 2, P_{k}$ est de classe $\mathcal{C}^{k-2}$ sur $\mathbb{R}$.

2) Pour tout $k \geq 1, P_{2 k+1}(0)=P_{2 k+1}(1)=0$.

3) Pour tout $k \geq 1, P_{2 k}(0)=P_{2 k}(1)=(-1)^{k-1} B_{k}$.

4) Pour tout $k \geq 1,\left|P_{2 k}(x)\right|$ est majoré par $B_{k}$.

Lemme 2. - Soit $f$ une fonction de classe $\mathcal{C}^{2}$ et intégrable sur $[m, \infty[$. On suppose en outre que la dérivée $f^{\prime}$ a une limite nulle à l'infini. Alors, pour tout entier $m \in \mathbb{Z}$ et tout entier $k \geq 1$ on a

$$
\begin{aligned}
\int_{m}^{+\infty} f(u) P_{2 k}(u) \mathrm{d} u= & \frac{(-1)^{k} B_{k+1}}{(2 k+1)(2 k+2)} f^{\prime}(m)+ \\
& +\frac{1}{(2 k+1)(2 k+2)} \int_{m}^{+\infty} f^{\prime \prime}(u) P_{2 k+2}(u) \mathrm{d} u
\end{aligned}
$$


Fonction sommatoire des inverses des termes d'une progression arithmétique

De plus, si $f^{\prime \prime}$ est de signe constant, dans le second membre de (9) le premier terme de la somme est plus grand que le second en valeur absolue. La somme est donc du signe du premier terme.

On en déduit le corollaire suivant.

Corollaire 1. - Si f est une fonction convexe de classe $\mathcal{C}^{2}$ intégrable sur $[m, \infty[$ et de dérivée nulle à l'infini alors

$$
\int_{m}^{+\infty} f(u) P_{2 k}(u) \mathrm{d} u
$$

est du signe de $P_{2 k}(0)$.

\section{Démonstration}

Le corollaire résulte du lemme 2 en remarquant que $(-1)^{k} f^{\prime}$ est du signe de $(-1)^{k-1}$ qui, par le lemme 1.3, est encore le signe de $P_{2 k}(0)$.

Démonstration du lemme 2.- Une première intégration par parties donne

$$
\begin{aligned}
\int_{m}^{+\infty} & f(u) P_{2 k}(u) \mathrm{d} u= \\
= & \frac{1}{2 k+1} \int_{m}^{+\infty} f(u) \mathrm{d}\left(P_{2 k+1}(u)\right) \\
= & \frac{1}{2 k+1}\left[P_{2 k+1}(u) f(u)\right]_{m}^{+\infty}-\frac{1}{2 k+1} \int_{m}^{+\infty} f^{\prime}(u) P_{2 k+1}(u) \mathrm{d} u \\
= & -\frac{1}{2 k+1} \int_{m}^{+\infty} f^{\prime}(u) P_{2 k+1}(u) \mathrm{d} u
\end{aligned}
$$

en remarquant que $P_{2 k+1}$ s'annule en $m$ car $k \geq 1$. Une deuxième intégration par parties donne alors :

$$
\begin{aligned}
& \int_{m}^{+\infty} f(u) P_{2 k}(u) \mathrm{d} u= \\
& =-\frac{1}{2 k+1} \int_{m}^{+\infty} f(u) \mathrm{d}\left(P_{2 k+1}(u)\right) \\
& =-\frac{1}{(2 k+1)(2 k+2)} \int_{m}^{+\infty} f^{\prime}(u) \mathrm{d}\left(P_{2 k+2}(u)\right) \\
& =\frac{-1}{(2 k+1)(2 k+2)}\left\{\left[P_{2 k+2}(u) f^{\prime}(u)\right]_{m}^{+\infty}-\int_{m}^{+\infty} f^{\prime \prime}(u) P_{2 k+2}(u) \mathrm{d} u\right\} \\
& =\frac{1}{(2 k+1)(2 k+2)}\left\{(-1)^{k} B_{k+1} f^{\prime}(m)+\int_{m}^{+\infty} f^{\prime \prime}(u) P_{2 k+2}(u) \mathrm{d} u\right\} .
\end{aligned}
$$


Si $f^{\prime \prime}$ est de signe constant, la dernière intégrale est majorée en valeur absolue par la valeur absolue de l'intégrale $\int_{m}^{+\infty} f^{\prime \prime}(u) B_{k+1} \mathrm{~d} u$ soit par $\left|B_{k+1} f^{\prime}(m)\right|$. La quantité entre accolades est donc du même signe que $(-1)^{k} B_{k+1} f^{\prime}(m)$.

\section{Quelques identités vérifiées par $J_{a, b}$}

On montre ici que l'étude de $J_{a, b}(x)$, défini par (7), se ramène à celle de $J_{a, 1}$. En effet, on a le résultat suivant.

LeMme 3.- On a les identités:

$$
\begin{aligned}
J_{a+b n, b}(x) & =J_{a, b}(x), \quad \forall n \in \mathbb{N}, \\
J_{a, b}(x) & =\frac{1}{b} J_{[a / b, 1]}\left(\frac{x}{b}\right) \\
J_{a, b}(x) & =\frac{1}{a} J_{[1, b / a]}\left(\frac{x}{a}\right) .
\end{aligned}
$$

Démonstration. - La première identité résulte de ce que $(u-a) / b$ et $(u-a-b n) / b$ ont la même partie fractionnaire. La deuxième identité s'obtient par le changement de variable $t=u / b$ dans l'intégrale (7), qui donne $J_{a, b}$, et la troisième par le changement de variable $t=u / a$.

Corollaire 2.- Pour démontrer le théorème 1 on peut supposer sans nuire à la généralité que $b=1$ et que $0<a \leq 1$.

Par (10) on peut, sans nuire à la généralité, supposer $a \leq b$ et supposant le théorème 1 vrai dans le cas où $b=1$, on a alors en utilisant (11) :

$$
J_{a, b}(x)=\frac{1}{b} J_{[a / b, 1]}\left(\frac{x}{b}\right)<\frac{1}{b} \frac{1}{24 x^{2} / b^{2}}=\frac{b}{24 x^{2}}
$$

et de même pour la minoration. 
Fonction sommatoire des inverses des termes d'une progression arithmétique

\section{Démonstration de la proposition 1}

Si $x \geq a$, en utilisant l'intégrale de Stieltjes, (3) devient :

$$
\begin{aligned}
A_{a, b}(x) & =\int_{a^{-}}^{x} \frac{\mathrm{d}\lfloor(u-a) / b\rfloor}{u} \\
& =\left[\frac{\lfloor(u-a) / b\rfloor}{u}\right]_{a^{-}}^{x}+\int_{a}^{x} \frac{\lfloor(u-a) / b\rfloor}{u^{2}} \mathrm{~d} u \\
& =\frac{\lfloor(x-a) / b\rfloor}{x}+\frac{1}{a}+\int_{a}^{x} \frac{\lfloor(u-a) / b\rfloor}{u^{2}} \mathrm{~d} u .
\end{aligned}
$$

Remplaçant $\lfloor t\rfloor$ par $t-\{t\}$, on obtient

$$
A_{a, b}(x)=\frac{\ln x}{b}+\gamma_{a, b}+\int_{x}^{\infty} \frac{\{(u-a) / b\}}{u^{2}} \mathrm{~d} u-\frac{\{(x-a) / b\}}{x}
$$

En remplaçant dans l'intégrale ci-dessus $\{(u-a) / b\}$ par

$$
\left\{\frac{u-a}{b}\right\}-\frac{1}{2}+\frac{1}{2}
$$

cela s'écrit encore

$$
A_{a, b}(x)=\frac{\ln x}{b}+\gamma_{a, b}-q_{a, b}(x)+J_{a, b}(x)
$$

et la proposition 1 est démontrée pour $x \geq a$.

Dans le cas $x<a$ on a

$$
\begin{aligned}
A_{a, b}(x) & =-\sum_{x<u<a}^{u \equiv a \bmod b} \frac{1}{u}=-\int_{x^{+}}^{a^{-}} \frac{\mathrm{d}\lfloor(u-a) / b\rfloor}{u} \\
& =-\left[\frac{\lfloor(u-a) / b\rfloor}{u}\right]_{x^{+}}^{a^{-}}-\int_{x}^{a} \frac{\lfloor(u-a) / b\rfloor}{u^{2}} \mathrm{~d} u \\
& =\frac{1}{a}+\frac{\lfloor(x-a) / b\rfloor}{x}+\int_{x}^{a} \frac{\lfloor(u-a) / b\rfloor}{u^{2}} \mathrm{~d} u .
\end{aligned}
$$

et on termine comme dans le cas $a \leq x$. 


\section{Formule d'Euler-MacLaurin de $J_{a, b}(x)$}

La fonction $J_{a, b}$ est continue sur $\mathbb{R}^{+}$. En un point $x$ qui n'est pas de la forme $a+b n, J_{a, b}$ a pour dérivée $(1 / 2-\{(x-a) / b\}) / x^{2}$. Chaque point $x$ de la forme $a+b n$ est un point anguleux, avec une dérivée à gauche égale à $-1 /\left(2 x^{2}\right)$, et une dérivée à droite égale à $1 /\left(2 x^{2}\right)$. Il en résulte que la fonction $J_{a, b}$ est oscillante avec un minimum en chaque point de la forme $a+b n$ et avec un maximum en chaque point de la forme $a+b n+b / 2$. $J_{a, b}(X)$ s'écrit :

$$
J_{a, b}(x)=\int_{x}^{\infty} \frac{P_{1}((u-a) / b)}{u^{2}} \mathrm{~d} u .
$$

Une intégration par parties donne alors, avec le lemme 1.1:

$$
J_{a, b}(x)=-\frac{b}{2 x^{2}} P_{2}\left(\frac{x-a}{b}\right)+b \int_{x}^{\infty} \frac{P_{2}((u-a) / b)}{u^{3}} \mathrm{~d} u .
$$

En répétant l'intégration par parties on obtient plus généralement :

$$
J_{a, b}(x)=-\sum_{k=2}^{k=r} \frac{b^{k-1}}{k x^{k}} P_{k}\left(\frac{x-a}{b}\right)+b^{r-1} \int_{x}^{\infty} \frac{P_{r}((u-a) / b)}{u^{r+1}} \mathrm{~d} u .
$$

Si dans cette formule on fait $r=3$ on obtient (en posant $u=\{(x-a) / b\})$ :

$$
J_{a, b}(x)=-\frac{b}{2 x^{2}}\left(u^{2}-u+\frac{1}{6}\right)-\frac{b^{2}}{3 x^{3}} P_{3}(u)+\mathrm{O}\left(\frac{1}{x^{3}}\right) .
$$

Si $x=a+b n, u=0$ et lorsque $n$ tend vers l'infini :

$$
J_{a, b}(x) \sim-\frac{b}{12 x^{2}} .
$$

Si $x$ tend vers l'infini avec $u=1 / 2$ on a

$$
J_{a, b}(x) \sim \frac{b}{24 x^{2}} .
$$

Il résulte de (16) et (17) que les constantes $-b / 12$ et $b / 24$ qui figurent dans le théorème 1 sont optimales. 
Fonction sommatoire des inverses des termes d'une progression arithmétique

\section{Minoration de $J_{a, b}(x)$}

Dorénavant on supposera que $b=1$ et $0<a \leq 1$, ce qui ne nuit pas à la généralité d'après le corollaire $2 \mathrm{du}$ lemme 3 . On posera $J(x)=J_{a, 1}(x)$ dans toute la suite.

LEMME 4. - En tout point $a_{n}=a+n, n \geq 0$, on $a$

$$
J\left(a_{n}\right) \geq-\frac{1}{12 a_{n}^{2}} .
$$

Démonstration. - On part de la formule (13) avec $b=1$ :

$$
J(x)=-\frac{1}{2 x^{2}} P_{2}(x-a)+\int_{x}^{+\infty} \frac{P_{2}(u-a)}{u^{3}} \mathrm{~d} u .
$$

Lorsque $x=a_{n}, x-a=n$ est un entier et $P_{2}(x-a)=1 / 6$. Par le corollaire du lemme 2 l'intégrale dans (18) est positive.

Proposition 2.- On a pour tout $a, x>0$ :

$$
-\frac{1}{12 x^{2}} \leq J(x) \text {. }
$$

Démonstration. - Il faut montrer que (19) reste vrai pour les $x$ qui ne sont pas de la forme $a+n$. Posons pour cela $g(x)=J(x)+1 /\left(12 x^{2}\right)$. Il faut montrer que la fonction $g(x)$ reste positive sur chaque intervale $\left[a_{n}, a_{n+1}\right]=[a+n, a+(n+1)]$ et aussi sur l'intervalle $\left.] 0, a\right]$. Or $g$ est dérivable sur ces intervalles, de dérivée

$$
\frac{1 / 2-\left(x-a_{n}\right)}{x^{2}}-\frac{1}{6 x^{3}}
$$

(Si l'intervalle considéré est l'intervalle ] $0, a$ ] on prend $a_{n}=a-1$.) Cette dérivée est du signe de

$$
x\left(a_{n}+\frac{1}{2}-x\right)-\frac{1}{6}
$$

qui prend la valeur $a_{n} / 2-1 / 6$ en $a_{n}$ et la valeur négative $-a_{n+1} / 2-1 / 6$ en $a_{n+1}$. On a alors : 
1) si $n \geq 1$ alors $a_{n}=a+n>1$ et la dérivée de $g$ est positive puis négative; le minimum de $g$ est donc atteint en l'un des deux points $a_{n}$ et $a_{n+1}$;

2) si $n=0$ :

- si $a \geq 1 / 3, g$ commence par croître, puis décroît sur [a, $a+1]$; le minimum de $g$ est donc atteint en $a_{0}$ ou en $a_{1}$;

- si $a<1 / 3, g$ décroît sur tout l'intervalle $[a, a+1]$; son minimum est atteint en $a+1$;

3) si l'intervalle considéré est l'intervalle $[0, a], g^{\prime}(x)$ est du signe de $x(a-1 / 2-x)-1 / 6$; ce trinôme atteint son maximum en $x=(a / 2-1 / 4)$ et ce maximum est

$$
\left(\frac{a}{2}-\frac{1}{4}\right)^{2}-\frac{1}{6}<0
$$

car $0<a \leq 1 ; g$ est donc décroissante sur ] $0, a$ ] et le minimum de $g$ atteint en $a$.

Le minimum absolu de $g$ est donc atteint en l'un des $a_{n}$ avec $n \geq 0$ et le lemme 4 achève la démonstration de la proposition 2 .

6. Une minoration de $h(x)=1 /\left(24 x^{2}\right)-J(x)$

Lemme 5. - Pour tout $n>0$, soit $x_{n}$ l'unique solution dans l'intervalle $\left[a_{n-1}, a_{n}\right]=[a+n-1, a+n]$ de l'équation

$$
\{x-a\}=\frac{1}{2}+\frac{1}{12 x}
$$

et soit $u_{n}=\left\{x_{n}-a\right\} ; u_{n}$ vérifie $1 / 2<u_{n}<2 / 3$. Sur l'intervalle $\left[a_{n-1}, a_{n}\right]$ la fonction $h(x)=1 /\left(24 x^{2}\right)-J(x)$ est décroissante de $a_{n-1} \grave{a}$ $x_{n}$ puis croissante de $x_{n}$ à $a_{n}$.

Démonstration. - La dérivée de $h$ est, pour $x$ non entier,

$$
h^{\prime}(x)=-\frac{1}{12 x^{3}}+\frac{1}{x^{2}}\left(\{x-a\}-\frac{1}{2}\right) ;
$$

elle est du signe de .

$$
\begin{gathered}
\left(\{x-a\}-\frac{1}{2}\right)-\frac{1}{12 x} \\
-480-
\end{gathered}
$$


Fonction sommatoire des inverses des termes d'une progression arithmétique

Sur l'intervalle $\left[a_{n-1}, a_{n}\right]$ cette quantité croît de $-1 / 2-1 /\left(12 a_{n-1}\right)$ jusqu'à $-1 / 2-1 /\left(12 a_{n}\right)$. Cette dernière quantité est positive car $a_{n}$ est au moins égal à $a+1$. La fonction $h$ commence donc par décroître sur l'intervalle $\left[a_{n-1}, a_{n}\right]$ puis croît à partir de la valeur $x_{n}$ solution de (20). Il résulte de (20) que $u_{n}=\left\{x_{n}-a\right\}=\left(x_{n}-a_{n-1}\right)$ vérifie $u_{n}>1 / 2$. Ceci entraîne

$$
x_{n}>\frac{1}{2}, \quad \frac{1}{12 x_{n}}<\frac{1}{6} \quad \text { et } \quad u_{n}<\frac{2}{3} .
$$

Lemme 6. - Reprenant les notations du lemme précédent : soit $n \geq 1$ et $x_{n}$ l'abscisse du minimum de $h$ sur l'intervalle $\left[a_{n-1}, a_{n}\right]$ et soit $u_{n}=\left\{x_{n}-a\right\}=\left(x_{n}-a_{n-1}\right)$. Alors, lorsque a décrit l'intervalle $\left.] 0,1\right]$,

1) $x_{1}=\frac{a}{2}+\frac{1}{4}+\frac{\sqrt{3} \sqrt{12 a^{2}+12 a+7}}{12}$

est une fonction croissante de a;

2) $u_{1}=x_{1}-a=-\frac{a}{2}+\frac{1}{4}+\frac{\sqrt{3} \sqrt{12 a^{2}+12 a+7}}{12}$ et $\frac{a+1}{x_{1}}$ sont des fonctions décroissantes de a;

3) $1 / x_{1}$ et $(a+1) / x_{1}$ sont majorés par

$$
\left(\frac{1}{4}+\frac{\sqrt{21}}{12}\right)^{-1}<1,583 ;
$$

4) si $n \geq 2,(a+n) / x_{n}<4 / 3$;

5) pour tout $n \geq 1,(a+n) / x_{n}<1,583$.

Démonstration. $-x_{n}$ est la racine positive de l'équation

$$
x=a+n-1+\frac{1}{2}+\frac{1}{12 x} \text {. }
$$

On en déduit l'expression de $x_{n}$

$$
x_{n}=\frac{1}{2} a+\frac{1}{2} n-\frac{1}{4}+\frac{\sqrt{3}}{12} \sqrt{12 a^{2}+24 n a-12 a+12 n^{2}-12 n+7} .
$$

En faisant $n=1$ on obtient alors successivement :

$$
\begin{aligned}
& x_{1}=\frac{a}{2}+\frac{1}{4}+\frac{\sqrt{3} \sqrt{12 a^{2}+24 a+7}}{12} \\
& u_{1}=x_{1}-a=-\frac{a}{2}+\frac{1}{4}+\frac{\sqrt{3} \sqrt{12 a^{2}+24 a+7}}{12}
\end{aligned}
$$




$$
\begin{aligned}
\frac{\mathrm{d} x_{1}}{\mathrm{~d} a} & =\frac{1}{2}+\frac{\sqrt{3}(24 a+12)}{24 \sqrt{12 a^{2}+12 a+7}}>0 \\
\frac{\mathrm{d} u_{1}}{\mathrm{~d} a} & =\frac{-\sqrt{12 a^{2}+12 a+7}+2 \sqrt{3} a+\sqrt{3}}{2 \sqrt{12 a^{2}+12 a+7}} \\
& =-\frac{\sqrt{12 a^{2}+12 a+7}-\sqrt{12 a^{2}+12 a+3}}{2 \sqrt{12 a^{2}+12 a+7}}<0
\end{aligned}
$$

$$
\begin{aligned}
& \frac{\mathrm{d}}{\mathrm{d} a}\left(\frac{a+1}{x_{1}}\right)= \\
& \quad=-12 \frac{3 \sqrt{12 a^{2}+12 a+7}+6 \sqrt{3} a-\sqrt{3}}{\left(6 a+3+\sqrt{3} \sqrt{12 a^{2}+12 a+7}\right)^{2} \sqrt{12 a^{2}+12 a+7}}<0 .
\end{aligned}
$$

Cela donne les deux premiers points. $1 / x_{1}$ et $(a+1) / x_{1}$ étant des fonctions décroissantes de $a$, leur maximum est atteint en $a=0$. Cela donne le 6.3. Par le lemme précédent, $x_{n}$ est supérieur à $a+n-1 / 2$ et, si $n \geq 2$ on a :

$$
\frac{a+n}{x_{n}} \leq \frac{a+n}{a+n-1 / 2}<\frac{n}{n-1 / 2} \leq \frac{4}{3}
$$

et cela termine la démonstration du quatrième point. Le cinquième résulte de 3 et 4 .

LEMME 7. - Soit $z_{4} \simeq 0,759$.. l'unique racine de $P_{4}(x)$ comprise entre 0,5 et 1 , et $x_{n}$ l'abscisse du minimum de $h$ sur l'intervalle $\left[a_{n-1}, a_{n}\right]$ (cf. lemme 5). $h\left(x_{n}\right)$ vérifie la minoration:

$$
\begin{aligned}
h\left(x_{n}\right) & =\frac{1}{24 x_{n}^{2}}-J\left(x_{n}\right) \geq \\
& \geq \frac{1}{x_{n}^{4}}\left[\frac{11}{2880}-\left(\int_{u_{n}}^{z_{4}} P_{4}(u) \mathrm{d} u-0,00049\right) \frac{1}{x_{n}}-\frac{7}{10368} \frac{1}{x_{n}^{2}}\right] .
\end{aligned}
$$

Démonstration. - Posons, comme dans le lemme $5, u_{n}=\left\{x_{n}-a_{n-1}\right\}=$ $x_{n}-\left(n-1\right.$. La formule (14) avec $k=4$ donne pour $J\left(x_{n}\right)$ la valeur

$$
J\left(x_{n}\right)=-\frac{1}{2 x^{2}} P_{2}\left(u_{n}\right)-\frac{1}{3 x_{n}^{3}} P_{3}\left(u_{n}\right)-\frac{1}{4 x_{n}^{4}} P_{4}\left(u_{n}\right)+\int_{x_{n}}^{+\infty} \frac{P_{4}(x-a)}{x^{5}} \mathrm{~d} x .
$$


Fonction sommatoire des inverses des termes d'une progression arithmétique

$\mathrm{Si}$, dans $(22)$, on substitue à $u_{n}$ sa valeur $u_{n}=1 / 2+1 /\left(12 x_{n}\right)$ tirée de (20), on obtient, à l'aide de Maple :

$$
\begin{aligned}
& J\left(x_{n}\right)=\frac{1}{24 x_{n}^{2}}-\frac{11}{2880 x_{n}^{4}}+\frac{7}{10368 x_{n}^{6}}-\frac{1}{82944 x_{n}^{8}}+\int_{x_{n}}^{+\infty} \frac{P_{4}(x-a)}{x^{5}} \mathrm{~d} u \\
& h\left(x_{n}\right)=\frac{11}{2880 x_{n}^{4}}-\frac{7}{10368 x_{n}^{6}}+\frac{1}{82944 x_{n}^{8}}-\int_{x_{n}}^{+\infty} \frac{P_{4}(x-a)}{x^{5}} \mathrm{~d} x .
\end{aligned}
$$

Effectuons dans l'intégrale le changement de variable $u=x-a-n+1$, cela donne

$$
\begin{aligned}
h\left(x_{n}\right)= & \frac{11}{2880 x_{n}^{4}}-\frac{7}{10368 x_{n}^{6}}+\frac{1}{82944 x_{n}^{8}}-\int_{u_{n}}^{+\infty} \frac{P_{4}(u)}{(a+n-1+u)^{5}} \mathrm{~d} u \\
\geq & \frac{11}{2880 x_{n}^{4}}-\frac{7}{10368 x_{n}^{6}}+ \\
& -\int_{u_{n}}^{1} \frac{P_{4}(u)}{(a+n-1+u)^{5}} \mathrm{~d} u-\int_{1}^{+\infty} \frac{P_{4}(u)}{(a+n-1+u)^{5}} \mathrm{~d} u .
\end{aligned}
$$

Par le corollaire 1 du lemme 2 , la dernière intégrale est négative et $h\left(x_{n}\right)$ est minoré par

$$
\begin{aligned}
& \frac{11}{2880 x_{n}^{4}}-\frac{7}{10368 x_{n}^{6}}-\int_{u_{n}}^{1} \frac{P_{4}(u)}{(a+n-1+u)^{5}} \mathrm{~d} u= \\
& =\frac{11}{2880 x_{n}^{4}}-\frac{7}{10368 x_{n}^{6}}-\int_{u_{n}}^{z_{4}} \frac{P_{4}(u)}{(a+n-1+u)^{5}} \mathrm{~d} u+ \\
& \quad+\int_{z_{4}}^{1} \frac{-P_{4}(u)}{(a+n-1+u)^{5}} \mathrm{~d} u .
\end{aligned}
$$

Entre $z_{4}$ et $1, P_{4}(u)$ est négatif, et $1 /(a+n-1+u)^{5}$ est minoré par $1 /(a+n)^{5}$, on a donc :

$$
\begin{aligned}
\int_{z_{4}}^{1} \frac{-P_{4}(u)}{(a+n-1+u)^{5}} \mathrm{~d} u & \geq \frac{1}{(a+n)^{5}} \int_{z_{4}}^{1}\left(-P_{4}(u)\right) \mathrm{d} u= \\
& =\frac{P_{5}\left(z_{4}\right)-P_{5}(1)}{5(a+n)^{5}} \geq \frac{0,00489}{(a+n)^{5}} \geq \\
& \geq \frac{0,00489}{1,583^{5} x_{n}^{5}} \geq \frac{0,00049}{x_{n}^{5}} \\
& -483-
\end{aligned}
$$


en utilisant le lemme 6 . Cela donne pour $h\left(x_{n}\right)$ la minoration :

$h\left(x_{n}\right) \geq \frac{1}{x_{n}^{4}}\left[\frac{11}{2880}+0,00049 \frac{1}{x_{n}}-\frac{7}{10368} \frac{1}{x_{n}^{2}}\right]+\int_{u_{n}}^{z_{4}} \frac{-P_{4}(u)}{(a+n-1+u)^{5}} \mathrm{~d} u$.

On en déduit (21) en minorant $(a+n-1+u)$ par $a+n-1+u_{n}=x_{n}$ sur l'intervalle $\left[u_{n}, z_{4}\right]$.

7. Minoration de $h(x)=1 /\left(24 x^{2}\right)-J(x) \operatorname{sur}[a,+\infty[$

Dans cette section on prouve la proposition suivante.

Proposition 3. - Soit $0<a \leq 1$. Alors on $a$

$$
J(x) \leq \frac{1}{24 x^{2}}
$$

pour tout $x \in[a,+\infty[$.

Démonstration. - On part de la minoration (21). Par le lemme 5,

$$
u_{n} \in\left[\frac{1}{2}, \frac{2}{3}\right] \subset\left[\frac{1}{2}, z_{4}\right] .
$$

Sur cet intervalle, $P_{4}$ prend des valeurs positives; on a donc

$$
\int_{u_{n}}^{z_{4}} P_{4}(u) \mathrm{d} u \leq \int_{1 / 2}^{z_{4}} P_{4}(u) \mathrm{d} u<0,00490 .
$$

Si on substitue ce majorant à l'intégrale dans la minoration (21) on obtient :

$$
h\left(x_{n}\right) \geq \frac{1}{x_{n}^{4}}\left[\frac{11}{2880}-0,00441 \frac{1}{x_{n}}-\frac{7}{10368} \frac{1}{x_{n}^{2}}\right] .
$$

Il en résulte que $h\left(x_{n}\right)$ est positif dès que $1 / x_{n}$ est plus petit que 0,774 . Il suffit pour cela que $x_{n}$ soit au moins égal à 1,3 . Si $n \geq 2$ cela est vérifié quel que soit $a$ car $x_{n}$ est au moins égal à $a+n-1 / 2>1,5$.

Pour terminer la démonstration on peut donc supposer $n=1$. Le lemme 6.1 montre que le rapport $1 / x_{1}$ est majoré par 0,774 si $a$ est minoré par 0,73 . On peut donc supposer $a<0,73$. Mais le 6.2 montre que sous cette 
Fonction sommatoire des inverses des termes d'une progression arithmétique

condition $u_{1}>0,564$. Cela permet d'améliorer la majoration de l'intégrale figurant dans (21) :

$$
\int_{u_{1}}^{z_{4}} P_{4}(u) \mathrm{d} u \leq \int_{0,564}^{z_{4}} P_{4}(u) \mathrm{d} u \leq 0,00307
$$

et, par (21), avec cette nouvelle majoration de l'intégrale :

$$
h\left(x_{1}\right) \geq \frac{1}{x_{1}^{4}}\left[\frac{11}{2880}-0,00258 \frac{1}{x_{1}}-\frac{7}{10368} \frac{1}{x_{1}^{2}}\right] .
$$

$h\left(x_{1}\right)$ est positif pourvu que le rapport $1 / x_{1}$ ne dépasse pas 1,14 Il suffit pour cela, par le lemme 6.1, que $a$ soit supérieur à 0,29 . On recommence en supposant $a<0,29$; d'où, par le lemme $6.2, u_{1}>0,594$ et

$$
\int_{u_{1}}^{z_{4}} P_{4}(u) \mathrm{d} u \leq \int_{0,594}^{z_{4}} P_{4}(u) \mathrm{d} u \leq 0,00229
$$

Cette nouvelle majoration et la formule (21) donnent une nouvelle minoration de $h\left(x_{1}\right)$ :

$$
h\left(x_{1}\right) \geq \frac{1}{x_{1}^{4}}\left[\frac{11}{2880}-0,00181 \frac{1}{x_{1}}-\frac{7}{10368} \frac{1}{x_{1}^{2}}\right]
$$

et ceci reste positif pourvu que $1 / x_{1}$ ne dépasse pas 1,39 . Il suffit pour cela, par le lemme 6.1, que $a<0,11$. On recommence une dernière fois en supposant $a<0,11$ d'où $u_{1}>0,614$ et

$$
\int_{u_{1}}^{z_{4}} P_{4}(u) \mathrm{d} u \leq \int_{0,614}^{z_{4}} P_{4}(u) \mathrm{d} u \leq 0,00181
$$

et

$$
h\left(x_{1}\right) \geq \frac{1}{x_{1}^{4}}\left[\frac{11}{2880}-0,00132 \frac{1}{x_{1}}-\frac{7}{10368} \frac{1}{x_{1}^{2}}\right]
$$

et le second membre est positif pourvu que $1 / x_{1}$ ne dépasse pas 1,59 , ce qui est toujours vérifié par le lemme 6.5. 
8. Minoration de $h$ sur $] 0, a[$

LeMme 8. - Soit h la fonction $x \mapsto 1 /\left(24 x^{2}\right)-J(x)$.

1) Si $0<a \leq 1 / 6, h$ est décroissante sur $] 0, a]$.

2) Si $1 / 6<a$, il existe un point $x_{0}(a)$ dans l'intervalle ] $\left.0, a\right]$ en lequel $h$ atteint son minimum sur $] 0, a]$. On note $u_{0}$ la partie fractionnaire de $x_{0}$. Alors :

(a) la valeur

$$
x_{0}(a)=\frac{a}{2}-\frac{1}{4}+\frac{\sqrt{3} \sqrt{12 a^{2}-12 a+7}}{12} ;
$$

(b) $u_{0}$ est une fonction décroissante de a;

(c) $x_{0}$ est une fonction croissante de $a$;

(d) $(a+1) / x_{0}$ est une fonction décroissante de $a$;

(e) $(a+1)^{4}>100 x_{0}^{4}$ pour tout $a>1 / 6$;

(f) soient $z_{1}<z_{2}$ les deux racines dans $[0,1]$ de $P_{2}(u)$; pour $a=1 / 2, u_{0}(a)=z_{2}$.

Démonstration. - Pour $x \in] 0, a],\{x-a\}=1+(x-a)$ et la dérivée de $h(x)$ sur l'intervalle ] $0, a$ ] est :

$$
\frac{1}{x^{2}}\left[\frac{1}{2}+x-a-\frac{1}{12 x}\right] \text {. }
$$

Le crochet croît de $-\infty$ à $1 / 2-1 /(12 a)$ sur l'intervalle ] $0, a]$. Si $a \leq 1 / 6$ le crochet est négatif pour tout $x$ de $] 0, a]$; la fonction $h(x)$ est donc minimum en $x=a$.

Supposons maintenant $1 / 6<a<1$. Dans ce cas la fonction $h$ passe par un minimum en le point $x_{0}$ de $] 0, a$ ] solution de

$$
x=a-\frac{1}{2}+\frac{1}{12 x} \text {. }
$$

La solution est

$$
x_{0}=\frac{a}{2}-\frac{1}{4}+\frac{\sqrt{3} \sqrt{12 a^{2}-12 a+7}}{12} .
$$


Fonction sommatoire des inverses des termes d'une progression arithmétique

La dérivée de $x_{0}$ est

$$
\begin{aligned}
\frac{\mathrm{d} x_{0}}{\mathrm{~d} a} & =\frac{\sqrt{12 a^{2}-12 a+7}+2 \sqrt{3} a-\sqrt{3}}{2 \sqrt{12 a^{2}-12 a+7}} \\
& \leq \frac{\sqrt{12 a^{2}-12 a+7}+\sqrt{12 a^{2}-12 a+3}}{2 \sqrt{12 a^{2}-12 a+7}} .
\end{aligned}
$$

Elle est positive et majorée par 1. La partie fractionnaire de $x_{0}-a$ est

$$
u_{0}=1+x_{0}-a=\frac{1}{12}+\frac{1}{12 x_{0}} .
$$

$u_{0}$ est donc décroissant.

Enfin la dérivée de $(a+1) / x_{0}$ est

$$
-12 \frac{9 \sqrt{12 a^{2}-12 a+7}+18 \sqrt{3} a-13 \sqrt{3}}{\left(6 a-3+\sqrt{3} \sqrt{12 a^{2}-12 a+7}\right)^{2} \sqrt{12 a^{2}-12 a+7}} .
$$

Le numérateur de la fraction est supérieur à $9 \sqrt{7}-13 \sqrt{3}$. La fonction $a \mapsto(a+1) / x_{0}$ est donc décroissante et atteint son minimum en $a=1$.

Lemme 9. - Soit $a>1 / 6$. Soit $x_{0}$ défini dans le lemme précédent comme l'abscisse du minimum de $h(x)$ sur l'intervalle ]0, a]. On a la majoration :

$$
\int_{2}^{+\infty} \frac{P_{2}(u)}{(a-1+u)^{3}} \mathrm{~d} u<\frac{1}{12000 x_{0}^{4}} .
$$

Démonstration. - Par le lemme 2 l'intégrale est positive majorée par

$$
\frac{1}{120(a+1)^{4}}<\frac{1}{12000 x_{0}^{4}}
$$

en utilisant le (e) du lemme 8.

Lemme 10. - Si $a>1 / 6$ et si $x_{0}$ est l'abscisse du minimun de $h$ sur l'intervalle ]0, $a$ ], on $a$ :

$$
\int_{u_{0}}^{2} \frac{P_{2}(u)}{(a-1+u)^{3}} \mathrm{~d} u \leq \frac{1}{300 x_{0}^{4}} .
$$




\section{Marc Deléglise}

Démonstration. - Pour démontrer (27) pour tous les $a$ de l'intervalle $[1 / 6,1]$ on va exhiber un ensemble d'intervalles $\left[a_{1}, a_{2}\right]$ qui recouvrent $[1 / 6,1]$ et sur chacun desquels (27) est vérifiée.

Sur l'intervalle $\left[a_{1}, a_{2}\right]$, par le lemme $8(\mathrm{c})$, on a en posant $x_{0}^{\prime \prime}=x_{0}\left(a_{2}\right)$ :

$$
\frac{1}{300 x_{0}^{4}}=\frac{1}{300\left(x_{0}(a)\right)^{4}} \geq \frac{1}{300\left(x_{0}^{\prime \prime}\right)^{4}} .
$$

La majoration du premier membre de (27) est plus fastidieuse, car le quotient

$$
\frac{P_{2}(u)}{(a-1+u)^{3}}
$$

est fonction croissante, ou décroissante de $a$ selon le signe de $P_{2}(u)$. Ceci nous oblige à découper l'intervalle d'intégration en intervalles sur lesquels $P_{2}$ garde un signe constant. $P_{2}(u)$ est positif sur $\left[0, z_{1}\right]$ et $\left[z_{2}, 1\right]$ et négatif $\operatorname{sur}\left[z_{1}, z_{2}\right]$.

Majoration de $\int_{u_{0}}^{2} \frac{P_{2}(u)}{(a-1+u)^{3}} \mathrm{~d} u$ pour $\left[a_{1}, a_{2}\right] \subset[1 / 6,0,5]$

Si $a \in\left[a_{1}, a_{2}\right]$ on a $a \leq 0,5$ et, par le lemme 8 (f), $u_{0} \geq z_{2}$; on écrit alors (après avoir fait le changement de variables $v=u-1$ dans les trois dernières intégrales) :

$$
\begin{aligned}
\int_{u_{0}}^{2} \frac{P_{2}(u)}{(a-1+u)^{3}} \mathrm{~d} u= & \int_{u_{0}}^{1} \frac{P_{2}(u)}{(a-1+u)^{3}} \mathrm{~d} u+\int_{0}^{z_{1}} \frac{P_{2}(v)}{(a+v)^{3}} \mathrm{~d} v+ \\
& +\int_{z_{1}}^{z_{2}} \frac{P_{2}(v)}{(a+v)^{3}} \mathrm{~d} v+\int_{z_{2}}^{1} \frac{P_{2}(v)}{(a+v)^{3}} \mathrm{~d} v .
\end{aligned}
$$

En partant de l'encadrement $a_{1} \leq a \leq a_{2}$, nous obtenons donc, en tenant compte des signes des fractions, la majoration

$$
\begin{aligned}
\int_{u_{0}}^{2} \frac{P_{2}(u)}{(a-1+u)^{3}} \mathrm{~d} u \leq & \int_{u_{0}}^{1} \frac{P_{2}(u)}{\left(a_{1}-1+u\right)^{3}} \mathrm{~d} u+\int_{0}^{z_{1}} \frac{P_{2}(u)}{\left(a_{1}+u\right)^{3}} \mathrm{~d} u+ \\
& +\int_{z_{1}}^{z_{2}} \frac{P_{2}(u)}{\left(a_{1}+u\right)^{3}} \mathrm{~d} u+\int_{z_{2}}^{1} \frac{P_{2}(u)}{\left(a_{1}+u\right)^{3}} \mathrm{~d} u .
\end{aligned}
$$


Fonction sommatoire des inverses des termes d'une progression arithmétique

La borne inférieure de la première intégrale, $u_{0}$ dépend de $a . \mathrm{Si}$ on pose $u_{0}^{\prime \prime}=u_{0}\left(a_{2}\right) \leq u_{0}$, on obtient enfin la majoration uniforme pour tous les $a$ de $\left[a_{1}, a_{2}\right]$ :

$$
\begin{aligned}
\int_{u_{0}}^{2} \frac{P_{2}(u)}{(a-1+u)^{3}} \mathrm{~d} u \leq & \int_{u_{0}^{\prime \prime}}^{1} \frac{P_{2}(u)}{\left(a_{1}-1+u\right)^{3}} \mathrm{~d} u+\int_{0}^{z_{1}} \frac{P_{2}(u)}{\left(a_{1}+u\right)^{3}} \mathrm{~d} u+ \\
& +\int_{z_{1}}^{z_{2}} \frac{P_{2}(u)}{\left(a_{2}+u\right)^{3}} \mathrm{~d} u+\int_{z_{2}}^{1} \frac{P_{2}(u)}{\left(a_{1}+u\right)^{3}} \mathrm{~d} u .
\end{aligned}
$$

Majoration de $\int_{u_{0}}^{2} \frac{P_{2}(u)}{(a-1+u)^{3}} \mathrm{~d} u$ pour $a \in\left[a_{1}, a_{2}\right] \subset[0,5,1]$

$\mathrm{Si}\left[a_{1}, a_{2}\right]$ est un intervalle contenu dans $[1 / 2,1]$, on pose $u_{0}^{\prime}=u_{0}\left(a_{1}\right)$ et on obtient de la même façon la majoration uniforme en $a$ :

$$
\begin{aligned}
\int_{u_{0}}^{2} & \frac{P_{2}(u)}{(a-1+u)^{3}} \mathrm{~d} u= \\
= & \int_{u_{0}}^{x_{2}} \frac{P_{2}(u)}{\left(a_{1}-1+u\right)^{3}} \mathrm{~d} u+\int_{z_{2}}^{1} \frac{P_{2}(u)}{(a-1+u)^{3}} \mathrm{~d} u+ \\
& \quad+\int_{0}^{z_{1}} \frac{P_{2}(u)}{(a+u)^{3}} \mathrm{~d} u+\int_{z_{1}}^{z_{2}} \frac{P_{2}(u)}{(a+u)^{3}} \mathrm{~d} u+\int_{z_{2}}^{1} \frac{P_{2}(u)}{(a+u)^{3}} \mathrm{~d} u .
\end{aligned}
$$

Le tableau suivant donne les majorants $M_{a_{1}, a_{2}}$ du rapport

$$
\int_{u_{0}}^{2} \frac{P_{2}(u)}{(a-1+u)^{3}} \mathrm{~d} u / \frac{1}{300 x_{0}^{4}}
$$

calculés par les formules ci-dessus, sur des intervalles [ $\left.a_{1}, a_{2}\right]$ qui recouvrent $[0,1]$. Tous ces majorants sont plus petits que 1 et cela termine la preuve du lemme 10.

Démonstration du théorème 1. - On sait déjà, par la proposition 3 que $h(x)=1 /\left(24 x^{2}\right)-J(x)$ est positive sur $[a,+\infty[$. Il reste à montrer que $h$ est positive sur $] 0, a]$. Si $a<1 / 6$, par le lemme $8.1, h$ est décroissante sur ] $0, a$ ]. Son minimum sur cet intervalle est atteint en $a$; il est positif puisque $h$ est positive sur $[a,+\infty]$. 


\begin{tabular}{|c|c|c|c|c|c|}
\hline$a_{1}$ & $a_{2}$ & $M_{a_{0}, a_{2}}$ & $a_{1}$ & $a_{2}$ & $M_{a_{1}, a_{2}}$ \\
\hline 0,16 & 0,20 & 0,715 & 0,20 & 0,24 & 0,872 \\
\hline 0,24 & 0,28 & 0,896 & 0,28 & 0,32 & 0,897 \\
\hline 0,32 & 0,36 & 0,885 & 0,36 & 0,40 & 0,864 \\
\hline 0,40 & 0,45 & 0,914 & 0,45 & 0,50 & 0,847 \\
\hline 0,50 & 0,55 & 0,833 & 0,55 & 0,60 & 0,826 \\
\hline 0,60 & 0,65 & 0,822 & 0,65 & 0,70 & 0,816 \\
\hline 0,70 & 0,75 & 0,809 & 0,75 & 0,80 & 0,800 \\
\hline 0,80 & 0,85 & 0,790 & 0,85 & 0,90 & 0,778 \\
\hline 0,90 & 1,00 & 0,460 & & & \\
\hline
\end{tabular}

On peut donc supposer $a>1 / 6$, et, puisque le minimum de $h$ sur ] $0, a$ ] est atteint en $x_{0}$, il faut montrer que $J\left(x_{0}\right)<1 /\left(24 x_{0}^{2}\right)$. La formule (13) donne :

$$
J\left(x_{0}\right)=-\frac{1}{2 x_{0}^{2}} P_{2}\left(1+x_{0}-a\right)+\int_{x_{0}}^{+\infty} \frac{P_{2}(1+x-a)}{x^{3}} \mathrm{~d} x .
$$

Par le changement de variable $u=1+x-a$ et par (26), cela donne :

$$
\begin{aligned}
J\left(x_{0}\right) & =\frac{1}{24 x_{0}^{2}}-\frac{1}{288 x_{0}^{4}}+\int_{u_{0}}^{+\infty} \frac{P_{2}(u)}{(a+u-1)^{3}} \mathrm{~d} u \\
& =\frac{1}{24 x_{0}^{2}}-\frac{1}{288 x_{0}^{4}}+\int_{u_{0}}^{2} \frac{P_{2}(u)}{(a-1+u)^{3}} \mathrm{~d} u+\int_{2}^{+\infty} \frac{P_{2}(u)}{(a-1+u)^{3}} \mathrm{~d} u .
\end{aligned}
$$

Par les lemmes 9 et 10 on a alors

$$
J\left(x_{0}\right)<\frac{1}{24 x_{0}^{2}}-\left(\frac{1}{288}-\frac{1}{300}-\frac{1}{12000}\right) \frac{1}{x_{0}^{4}}=\frac{1}{24 x_{0}^{2}}-\frac{1}{18000 x_{0}^{4}}<\frac{1}{24 x_{0}^{2}}
$$

qui termine la preuve du théorème 1.

9. Calcul numérique de $J_{a, b}(x)$ et de la constante $\gamma_{a, b}$

Bien que ceci ne soit pas utile à la démonstration du théorème 1 , donnons dans cette section quelques indications sur le calcul numérique 
Fonction sommatoire des inverses des termes d'une progression arithmétique

de la constante $\gamma_{a, b}$ et de $J_{a, b}$. Si dans la formule (14) on prend pour $x$ un nombre de la forme $a+b m$ et pour $r$ un entier pair $r=2 p$ on obtient :

$$
\begin{aligned}
J(a+b m)= & -\sum_{k=1}^{k=p} \frac{b^{2 k-1}}{2 k(a+b m)^{2 k}} P_{2 k}(0)+ \\
& +b^{2 p-1} \int_{a+b m}^{\infty} \frac{P_{2 p}((u-a) / b)}{u^{2 p+1}} \mathrm{~d} u \\
= & \sum_{k=1}^{k=p} \frac{(-1)^{k} b^{2 k-1}}{2 k(a+b m)^{2 k}} B_{k}+b^{2 p-1} \int_{a+b m}^{\infty} \frac{P_{2 p}((u-a) / b)}{u^{2 p+1}} \mathrm{~d} u \\
= & \sum_{k=1}^{k=p} \frac{(-1)^{k} b^{2 k-1}}{2 k(a+b m)^{2 k}} B_{k}+b^{2 p} \int_{m}^{\infty} \frac{P_{2 p}(v)}{(a+b v)^{2 p+1}} \mathrm{~d} v
\end{aligned}
$$

et, par le lemme 2 , la dernière intégrale est du signe de

$$
\frac{(-1)^{p+1} b^{2 p+1}}{2(p+1)(a+b m)^{2 p+2}} B_{p+1}
$$

et majorée en valeur absolue par cette fraction. Autrement dit pour tout entier positif $p$ il existe un réel $\lambda$ compris entre 0 et 1 tel que :

$$
J_{a, b}(a+b m)=\sum_{k=1}^{k=p} \frac{(-1)^{k} b^{2 k-1}}{2 k(a+b m)^{2 k}} B_{k}+\lambda \frac{(-1)^{p+1} b^{2 p+1}}{2(p+1)(a+b m)^{2 p+2}} B_{p+1} .
$$

Pour calculer $J_{a, b}(x)$ on choisit donc un entier $m$ tel que $a+b m$ soit supérieur à $x$ et pas trop petit, et on écrit

$$
J_{a, b}(x)=\int_{x}^{a+b m} \frac{\{(u-a) / b\}-1 / 2}{u^{2}} \mathrm{~d} u+J_{a, b}(a+b m) .
$$

On calcule l'intégrale directement et $J_{a, b}(a+b m)$ par (28).

Pour calculer $\gamma_{a, b}$ la proposition 1 et l'égalité (28) donnent :

$$
\gamma_{a, b}=\sum_{k=0}^{k=p} \frac{1}{a+b k}-\frac{\ln (a+b m)}{b}-\frac{1}{2(a+b m)}+\sum_{k=1}^{k=p} \frac{(-1)^{k-1} b^{2 k-1}}{2 k x^{2 k}} B_{k}
$$


l'erreur commise étant du signe du premier terme négligé et majorée en valeur absolue par celui-ci. On donne ici les premiers termes de ce développement :

$$
\begin{aligned}
\gamma_{a, b}= & \sum_{k=0}^{k=p} \frac{1}{a+b k}-\frac{\ln (a+b m)}{b}-\frac{1}{2(a+b m)}+\frac{b}{12(a+b m)^{2}}+ \\
& -\frac{b^{3}}{120(a+b m)^{4}}-\frac{b^{5}}{252(a+b m)^{6}}+\frac{b^{7}}{240(a+b m)^{8}}+ \\
& +\frac{b^{9}}{132(a+b m)^{10}}-\frac{691 b^{11}}{32760(a+b m)^{12}}+ \\
& +\frac{b^{13}}{12(a+b m)^{14}}-\frac{3617 b^{15}}{8160(a+b m)^{16}}+\cdots
\end{aligned}
$$

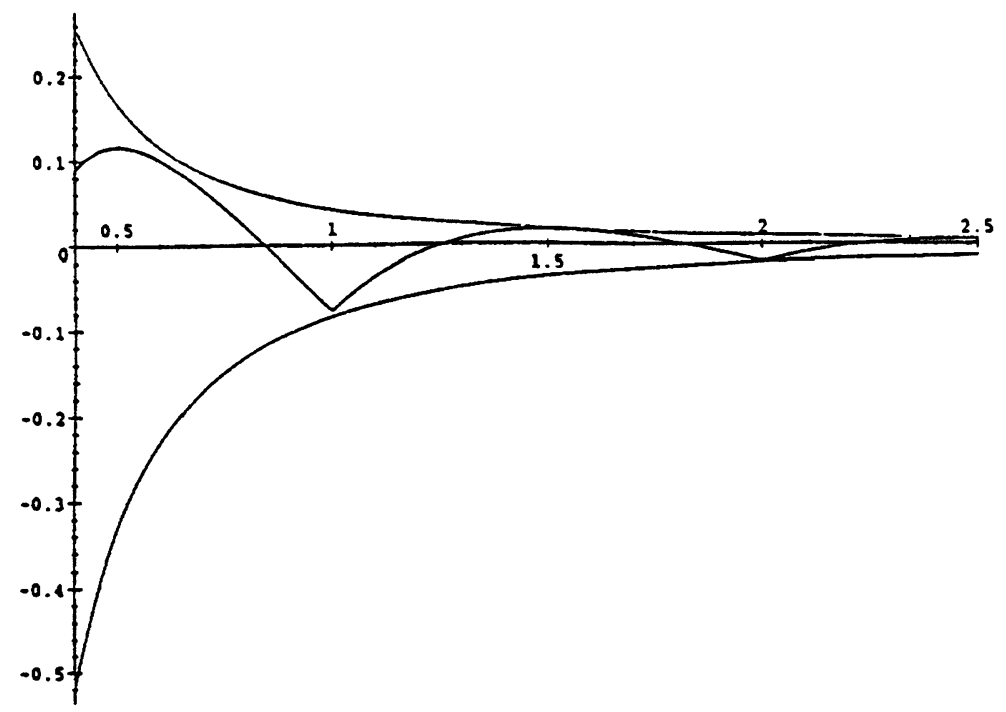

Fig. 1 Graphes de $J(x),-1 /\left(12 x^{2}\right)$ et $1 /\left(24 x^{2}\right)$ pour $a=b=1$ et $0,4 \leq x \leq 2,5$.

\section{Bibliographie}

[1] Hardy (G. H.) - - Divergent Series, Clarendon Press, Oxford, 1962.

[2] Lehmer (D. H.) .- Euler constants for arithmetical progressions, Acta Arithmetica XXVII (1975), pp. 125-142. 